Research Letter

\title{
SEROPREVALENCE AND CLINICAL SPECTRUM OF SARS-CoV-2 INFECTION IN THE FIRST VERSUS THIRD TRIMESTER OF PREGNANCY
}

Francesca Crovetto, $\mathrm{MD}, \mathrm{PhD}^{1}$, Fàtima Crispi, $\mathrm{MD}, \mathrm{PhD}^{1}$, Elisa Llurba, $\mathrm{MD}, \mathrm{PhD}^{2,3}$, Francesc Figueras, $\mathrm{MD}, \mathrm{PhD}^{1}$, María Dolores Gómez-Roig, $\mathrm{MD}, \mathrm{PhD}^{1,3}$, Eduard Gratacós, $\mathrm{MD}, \mathrm{PhD}^{1}$ *.

${ }^{1}$ BCNatal - Barcelona Center for Maternal-Fetal and Neonatal Medicine (Hospital Sant Joan de Déu and Hospital Clínic), Institut de Recerca Sant Joan de Déu, IDIBAPS, Universitat de Barcelona, Center for Biomedical Network Research on Rare Diseases, Barcelona, Spain.

${ }^{2}$ Obstetrics and Gynaecology Department, Sant Pau University Hospital, Universitat Autònoma de Barcelona, Barcelona, Spain.

${ }^{3}$ Maternal and Child Health and Development Network (SAMID), RD16/0022/0015, Instituto de Salud Carlos III, Spain.

Word count: 585

*Corresponding author: Eduard Gratacós, Head and Professor, BCNatal (Hospital Sant Joan de Déu and Hospital Clínic), Universitat de Barcelona, Passeig de Sant Joan de Déu 2, 08950, Esplugues de Llobregat, Barcelona, Spain. E-mail: egratacos@sjdhospitalbarcelona.org

\section{Introduction}

Case registries of pregnant women diagnosed with coronavirus disease (COVID-19) by polymerase chain reaction (PCR) have reported that the majority experienced mild infection, but up to $9 \%$ may require critical care. ${ }^{1}$ Most COVID-19 cases published were in the third trimester of pregnancy, which could reflect reporting bias, higher risk of infection or increased disease severity in late pregnancy. ${ }^{2}$ Seroprevalence studies may allow reliable estimates of the susceptibility to infection and clinical spectrum since they include asymptomatic and mild infections not tested for PCR. We evaluated the seroprevalence and clinical presentation of severe acute respiratory syndrome coronavirus 2 (SARS-CoV-2) infection in pregnant women in the first and third trimester.

\section{Methods}

The study was approved by the Institutional Review Board at each institution and informed consent was obtained. We recruited 874 consecutive pregnancies attending for first trimester screening (10-16 weeks' gestation, $n=372)$ or delivery $(n=502)$ from April 14 to May 5. All women were interviewed with a structured questionnaire for COVID-19 symptoms two months prior to sampling. SARS-CoV-2 IgG and IgM/IgA antibodies were tested (COVID19 VIRCLIA® Monotest, Vircell Microbiologist, Spain; reported sensitivity $70 \%$ IgG and $89 \% \mathrm{IgM} / \operatorname{IgA}$, and specificity $89 \%$ and $99 \%$ respectively). Indeterminate results were retested (VITROS $®$ Immunodiagnostic Products Anti-SARS-CoV2 Total Tests, Ortho Clinical Diagnostics, USA; $100 \%$ sensitivity and specificity) and re-classified as positive or negative. Women with COVID-19 were diagnosed and managed according to standard protocols and guidelines $^{3,4}$. Statistical differences were tested using the $\chi^{2}$ test or Student t-test as appropriate $(\mathrm{p}<0.05)$. 


\section{Results}

A total of 125 of 874 women (14.3\%) were positive for either IgG or IgM/IgA SARS-CoV-2 antibodies, 54/372 (14.5\%) in the first and 71/502 (14.1\%) in the third trimester. A total of $75 / 125(60 \%)$ reported no symptoms of COVID-19 in the past 2 months, whereas $44(35.2 \%)$ reported one or more symptoms, of which $31(24.8 \%)$ had at least 3 symptoms or anosmia and $8(6.4 \%)$ dyspnea. Overall, 7 women $(5.6 \%)$ were admitted for persistent fever $\left(>38^{\circ}\right)$ despite paracetamol and dyspnea, of which 3 had signs of pneumonia on chest radiography. All 3 had criteria for severity (bilateral chest condensation, respiratory rate>30 and leukopenia) and required oxygen support but not critical care or mechanical ventilation, and they were all discharged well. The rates of symptomatic infection, hospital admission or dyspnea were significantly higher in third trimester women (Table and Figure).

\section{Discussion}

The $14.3 \%$ seroprevalence of SARS-COV-2 in pregnant women in this study was substantially larger than the contemporary rates of PCR positive cases $(0.78 \%)$ reported for women 20-40y in Barcelona. ${ }^{5}$ The data confirm that COVID-19 is asymptomatic in the majority of pregnant women ${ }^{6}$ and illustrate the value of seroprevalence studies to capture the high proportion of asymptomatic or mild infections. In this study, none of the 125 pregnant women with SARS-CoV-2 infection required critical care as compared to $9 \%$ reported in cases diagnosed with PCR. ${ }^{1}$ However, the proportion of infections with symptoms or dyspnea was remarkably higher in the third trimester, and these results are in line with COVID-19 registries, reporting that $81 \%$ of hospitalized women were in late pregnancy or peripartum. ${ }^{1}$

These results provide reassuring information that, even in settings with a high prevalence, SARS-CoV-2 infection in pregnancy mostly presents with asymptomatic or mild clinical forms. The susceptibility to infection seemed to be the same in the first and the third trimesters of gestation. The data further suggest that, as with other respiratory viruses, COVID-19 could be more severe and require increased surveillance in late pregnancy. These findings should be confirmed and extended with larger consecutive prevalence studies in pregnancy.

Funding: This study was funded by the KidsCorona Child and Mother COVID-19 Open Data \& Biobank Initiative, Hospital Sant Joan de Déu, and "LaCaixa" Foundation, Barcelona, Spain.

Additional contributions: We thank all the medical staff, residents, midwifes and nurses of BCNatal (Hospitals Sant Joan de Déu and Clínic) and Hospital Sant Pau, especially Rosalia Pascal, MD, Marta Larroya, MD, Cristina Trilla, MD, Martí Cantallops, MD, Marta Camacho, MD, M. Carmen Medina, MD, Irene Casas, MD, Marta Tortajada, MD, Àlex Cahuana, MD, Patricia Muro, MD, Marta Valdés, MD, David Boada, MD and Anna Mundo, $\mathrm{MD}$, for careful data collection; Angela Arranz, $\mathrm{PhD}$, for her key role in the coordination of the nursing research team; Imma Mercade, $\mathrm{PhD}$, Elena Casals, $\mathrm{PhD}$, and Josefina Mora, $\mathrm{PhD}$, for their contribution in the collection of first trimester samples; Jordi Yague, $\mathrm{PhD}$, and $\mathrm{M}$. Angeles Marcos, $\mathrm{PhD}$, for supervising the antibodies analyses; and the Biobanks of Fundació Sant Joan de Déu, Clínic-IDIBAPS and Sant Pau for valuable management of samples. None of them received any compensation for their contribution.

\section{References}


1. The UK Obstetric Surveillance System SARS-CoV-2 Infection in Pregnancy Collaborative Group. Characteristics and outcomes of pregnant women hospitalised with confirmed SARS-CoV-2 infection in the UK: a national cohort study using the UK Obstetric Surveillance System (UKOSS). medRxiv preprint doi: https://doi.org/10.1101/2020.05.08.20089268

2. Rasmussen SA, Jamieson DJ, Bresee JS. Pandemic influenza and pregnant women. Emerg Infect Dis 2008;14:95-100.

3. López M, Gonce A, Meler E, et al. COVID-19 in pregnancy: a clinical management protocol and considerations for practice. Fetal Diagn Therapy 2020. http://doi.org/10.1159/000508487

4. Metlay JP, Waterer GW, Long AC, Anzueto A, Brozek J, Crothers K, et al. Diagnosis and treatment of adults with community-acquired pneumonia. Am J Respir Crit Care Med. 2019;200:E45-67.

5. http://aquas.gencat.cat/ca/actualitat/ultimes-dades-coronavirus/mapa-permunicipis/index.html\#googtrans(calen)

6. Breslin N, Baptiste C, Gyamfi-Bannerman C, et al. Coronavirus disease 2019 among asymptomatic and symptomatic pregnant women: two weeks of confirmed presentations to an affiliated pair of New York City hospitals. Am J Obstet Gynecol MFM 2020 Apr 9;X100118. http://doi.org/10.1016/j.ajogmf.2020.100118 
Table. Baseline characteristics and clinical features of pregnant women positive for SARS-CoV-2 infection $(\mathrm{N}=125)$ in the first versus third trimester.

\begin{tabular}{lccc}
\hline \multicolumn{1}{c}{ Characteristics } & $\begin{array}{c}\text { First } \\
\text { Trimester }\end{array}$ & $\begin{array}{c}\text { Third } \\
\text { Trimester }\end{array}$ & p \\
& N=54 & N=71 & \\
\hline Age (years) & $33.7(5.1)$ & $31.8(6.1)$ & 0.062 \\
White ethnicity & $31(57.4)$ & $43(60.6)$ & 0.697 \\
Body mass index $\left(\mathrm{Kg} / \mathrm{m}^{2}\right)$ & $24.1(6.2)$ & $23.9(4.3)$ & 0.209 \\
Smoking status & $4(7.4)$ & $6(8.5)$ & 0.543 \\
Medical history & & & \\
$\quad$ Chronic hypertension & $1(1.9)$ & $2(2.8)$ & 0.602 \\
$\quad$ Diabetes mellitus & $1(1.9)$ & $0(0)$ & 0.432 \\
$\quad$ Autoimmune disease & $2(3.7)$ & $0(0)$ & 0.185 \\
$\quad$ Respiratory disease & $2(3.7)$ & $3(4.2)$ & 0.297 \\
Nulliparous & $34(63)$ & $36(50.7)$ & 0.095 \\
Multiple pregnancy & $2(3.7)$ & $1(1.4)$ & 0.398 \\
\hline Symptoms compatible with COVID-19 within last 2 & & & \\
months & & & \\
$\quad$ None & $38(70.4)$ & $37(52.1)$ & 0.012 \\
$\quad$ Fever & $7(13)$ & $16(22.5)$ & 0.152 \\
$\quad$ Dry cough & $5(9.3)$ & $17(23.9)$ & 0.034 \\
$\quad$ Dyspnea & $0(0)$ & $8(11.3)$ & 0.010 \\
Tiredness & $5(9.3)$ & $4(5.6)$ & 0.307 \\
Sore throat & $6(11.1)$ & $3(4.2)$ & 0.116 \\
Rash on skin, or discoloration of fingers/toes & $0(0)$ & $0(0)$ & $\mathrm{NA}$ \\
Diarrhea & $4(7.4)$ & $5(7)$ & 0.575 \\
Loss of taste or smell & $7(13)$ & $16(22.5)$ & 0.152 \\
At least 3 symptoms or anosmia & $9(16.7)$ & $22(31.0)$ & 0.065 \\
$\quad$ Fever, cough and dyspnea & $0(0)$ & $8(11.3)$ & 0.010 \\
Hospital admission for COVID-19 symptoms & $0(0)$ & $7(9.9)$ & 0.019 \\
\hline
\end{tabular}

SARS-CoV-2: severe acute respiratory syndrome coronavirus 2

Data are expressed as n (\%) or mean (SD) 
medRxiv preprint doi: https://doi.org/10.1101/2020.06.17.20134098; this version posted June 19, 2020. The copyright holder for this preprint (which was not certified by peer review) is the author/funder, who has granted medRxiv a license to display the preprint in perpetuity.

All rights reserved. No reuse allowed without permission.

Figure. Clinical spectrum of SARS-CoV-2 infection in pregnant women in the first and third trimester.
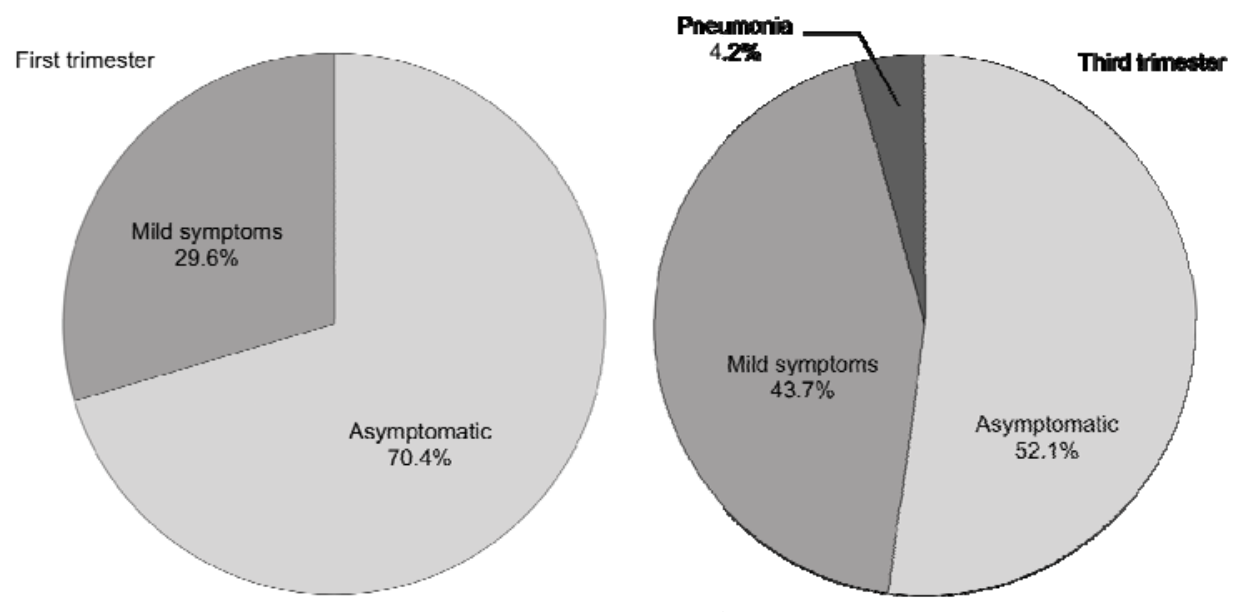